\title{
RECENT X-RAY OBSERVATIONS OF SUPERNOVA REMNANTS AND THEIR INTERPRETATION
}

\author{
B. Aschenbach \\ Max-Planck-Institut für Physik und Astrophysik \\ Institut für extraterrestrische Physik \\ 8046 Garching, W-Germany
}

\begin{abstract}
A review is given of recent observations of the $\mathrm{X}$ ray emission from supernova remnants carried out on the Einstein, Tenma and EXOSAT satellites as well as from a few sounding rocket experiments. Our current interpretation of the high resolution images, high resolution energy spectra and the first few spectrally resolved images is discussed.
\end{abstract}

Introduction: Almost five years ago, in 1982 the IAU Symposium No. 101 on "Supernova Remnants and their X-ray Emission" was held in Venice. The proceedings edited by Danziger and Gorenstein include an extensive compilation of papers dealing with observations mainly made with the instruments on board of the Einstein satellite. It was in fact the Einstein observatory with its imaging and spectroscopic capability through which substantial progress has been made in supernova remnant research. Since then further contributions have come from the EXOSAT observatory of the European Space Agency; a preliminary account of which has been given by Aschenbach (1985), the Japanese Tenma satellite and a few sounding rocket experiments. Unlike radio and optical observations X-ray observations can be done exclusively from space and therefore substantial observational research is limited to the rare opportunities of X-ray astronomy satellites. Einstein, EXOSAT and Tenma terminated operation and the next block of observations will not come before 1990, when the German satellite ROSAT will be launched.

As of today there are about 150 galactic supernova remnants catalogued which have been identified as such by their radio morphology and their radio spectrum. From 40 of these objects X-ray emission has been detected. Thus the vast majority of the alleged supernova remnants have been seen only at radio frequencies and their true nature has still to be explored by future more sensitive observations. Outside the Galaxy 32 remnants have been found in the Large Magellanic Cloud and 6 in the Small Magellanic Cloud (respectively Mathewson et al., 1985 and Helfand, 1987). $\mathrm{X}$-ray instrumentation has by now evolved to such a state that apart from this pure statistical information, which is however relevant to estimate supernova rates and supernova remnant lifetimes for instance, detailed studies of images, energy spectra and time variability of individual remnants can be made.

Images: High quality images became available first from the Einstein observatory with an angular resolution of typically 10 arcsec. The brighter remnants have subsequently been imaged by the smaller EXOSAT telescopes with poorer resolution. These images have been used to support the view of at least two main classes of remnants, which are the Crab-like rem- 
nants and the shell-type remnants. In his review Seward (1985) defines the Crab-like remnants as extended objects with a non-thermal X-ray spectrum with some evidence for a central energy source like an active pulsar. Crab-like remnants may appear as filled shells ("plerions") or as composite remnants which show both a radio shell and diffuse or point-like emission from the central region (Weiler, 1983). The recent EXOSAT observations have been presented by Davelaar and Smith (1986). Clearly, this class of remnants is intimately connected to neutron star research and a review on this topic has been given by Helfand and Becker (1984). Nomoto and Tsuruta (1986) have summarized the available data and upper limits on brightness and surface temperature of neutron stars in supernova remnants and have confronted it with current cooling theories. Clearly, more relevant in the context of this IAU Colloquium on the interaction of supernova remnants with the interstellar medium is the second class of remnants which is that of shells with a thermal spectrum. Unlike the Crab-like remnants which are most likely energized by a central compact object (Pacini and Salvati 1973, Rees and Gunn 1974, Aschenbach and Brinkmann 1975, Reynolds and Chevalier 1984, Brinkmann et al. 1986), the shell type remnants emit radiation from an optically thin plasma, which has been heated to Xray temperatures by a shock wave. This shock wave may either be the blast wave associated with the stellar explosion (Heiles 1964), which interacts with the ambient interstellar or circumstellar material, or it may be a reverse shock wave (McKee 1974), which propagates inwards from the decelerated blast wave and raises the temperature of the stellar ejecta. In both cases the X-ray emitting shell (or shells) is expected to be perfectly circular and to have no spatial structure in surface brightness except a radial gradient for a spherically symmetrical explosion and homogeneous media. The X-ray images, however, have revealed quite the opposite with a great deal of structure present in all remnants. The young remnants Cas-A, Kepler, Tycho and SN1006 are still maintaining an approximate circular shape whereas older ones like Puppis-A are hard to reconcile with a shell at all (see figure 1).

Since X-ray emission scales with the square of density it is obvious to consider density variations within or across the extent of the remnant as the dominant source for the observed X-ray brightness structures. Large scale uniform density changes like the interstellar density gradient perpendicular to the galactic plane have been made responsible for the asymmetry in galactic latitude, observed in Puppis-A for instance (Petre et al. 1982). The increased density towards the galactic plane as well as, to a a smaller extent, the increased slow-down of the blast wave, gives rise to to higher X-ray surface brightness in the same direction. If a remnant has grown to such a size the blast wave has passed quite a number of individual cool, high density interstellar clouds of different sizes, as well as their warm, medium density envelopes, which are embedded in the general hot, low density intercloud medium (McKee 1981), and these structures will directly be imaged in X-rays if heated to the required temperatures. So, the interstellar medium structure will be visible in the $X$-ray images of relatively old remnants. Younger remnants show brightness variations as well, which are attributed to the clumpiness of the stellar ejecta heated by the reverse shock. 


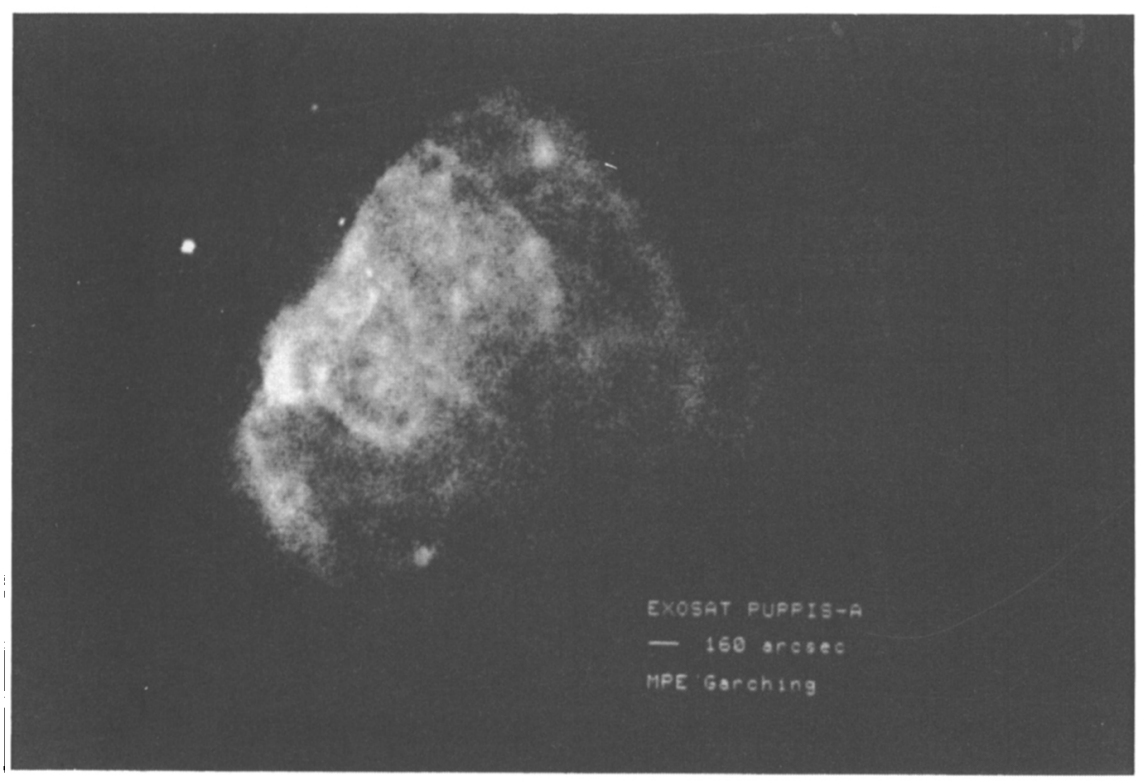

Fig. 1. EXOSAT soft X-ray image of Puppis-A.

The detailed X-ray appearance of clouds in a low density medium depends on the physics of the interaction with the shock wave. Cowie and McKee (1977) and McKee (1981) have considered mass evaporation and hydrodynamic ablation to increase the density in the adjacent intercloud medium, thus raising the $X$-ray emission. An alternative approach to increase the $X$ ray emission near dense clouds has been proposed by Hester and Cox (1986). Led by an analysis of the X-ray and optical emission of the Cygnus Loop, they have proposed an additional compression of the down-stream Xray emitting plasma by reflected or bow shocks around dense clouds. High resolution X-ray images of such clouds and their surroundings will help to discriminate the two effects, in particular if X-ray temperatures can be determined, which are supposed to be higher in the bow shock model. It is interesting to note (McKee 1981), that evaporation dominated remnants are affected on a large scale by the mass transfer from the clouds to the intercloud medium. In this case pressure and density will increase radially inwards contrary to the classical Sedov solution. Finally, non-thermal contributions to the X-ray emission may be not negligible in regions of electron acceleration and magnetic field amplification associated with the shock waves (Reynolds and Chevalier 1981).

X-ray images have been compared with optical and radio images, and the images of an individual remnant usually match each other quite well. In particular, the radio and X-ray images show very similar shapes, the outermost contours of which agree in remarkable detail down to the angular resolution limit. This indicates that heating of the plasma to X-ray energies and acceleration of electrons and magnetic field amplification sufficient for radio synchrotion radiation occur at least close to the shock front of the blast wave and on about the same time scale. Within the remnant the correlation is often less good. Radio bright regions sometimes coincide with 
X-ray bright knots, but are more often slightly offset as in Puppis-A (Petre et al. 1982). In the majority X-ray enhancements have no radio counterpart and vice versa. The same picture holds for the comparison of $X$ ray and optical images, which are dominated by filamentary and knotty structure. The fact that some optically bright filaments are embedded in $\mathrm{X}$-ray bright regions, for instance in the Vela remnant (Kahn et al. 1985) and in the Cygnus Loop (Hester and Cox 1986), indicates that the optical emission is due to dense shocked clouds, which either partly evaporate or produce bow shocks to enhance the ambient X-ray emission. Beyond this the comparison of images taken in the different spectral domains has so far not provided deeper insights in the physics of supernova remnants, despite the high angular resolution now available in $\mathrm{X}$-ray images.

Energy spectra: The thermal origin of the emission from shell type supernova remnants was for the first time established unambiguously by the detection of the Fe-K emission line in the spectrum of Cas-A (Serlemitsos et al. 1973). However, the clear detection of additional emission lines at lower energies from other atomic species was severely hampered by the low spectral resolution of the collimated proportional counter detectors. This changed with the advent of the solid state spectrometer (SSS) which was used in the focus of the Einstein observatory X-ray telescope. With an improved resolution of about $160 \mathrm{eV}$ over the nominal 0.5-4.5 keV detection band, numerous emission lines from highly ionized $\mathrm{Mg}, \mathrm{Si}, \mathrm{S}, \mathrm{Ar}$, and $\mathrm{Ca}$ have been measured in the spectra of young supernova remnants including Cas-A, Kepler and Tycho (Holt 1983). Additional lines from highly ionized $\mathrm{N}, \mathrm{O}$ and $\mathrm{Ne}$, as well as from $\mathrm{Fe}$ XVII have been discovered with the Einstein Bragg Focal Plane Crystal Spectrometer (FPCS) in the older remnant Puppis-A. With a resolving power of 100-1000, the forbidden, intercombination and recombination lines of the He-like triplets from $O$ VII and $\mathrm{Ne}$ IX have been resolved for the first time (Winkler et al. 1981). The spectral survey of emission lines from Puppis-A, which is the most detailed result of non-solar X-ray spectroscopy so far, is displayed in figure 2 . Winkler et al. (1983) have pointed out in a very clear way how the various line ratios can be used to perform detailed plasma diagnostics in determining electron temperature, ion population, ionization temperature and ionization time.
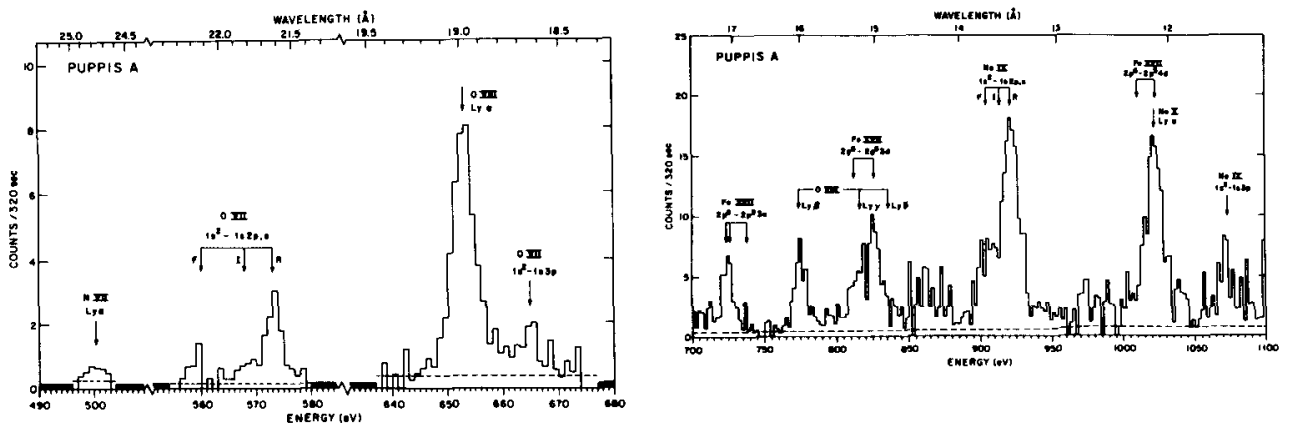

Fig. 2. Einstein FPCS spectra of Puppis-A (from Winkler et al. 1981).

The Einstein energy band extended up to about $4.5 \mathrm{keV}$ and therefore the 
Fe-K line emission as well as the high energy continuum, the existence of which was known from previous experiments, could not be covered simultaneously. Energy spectra over the $2-10 \mathrm{keV}$ range with a spectral resolution better than the collimated proportional counter by typically a factor of 2 have become available from the non-imaging gas scintillation proportional counters (GSPC) flown on board of the EXOSAT and Tenma satellites. Figure 3 shows the EXOSAT GSPC spectrum of Tycho (Smith et al. 1987). The emission lines from transitions of He-like $\mathrm{S}, \mathrm{Ar}, \mathrm{Ca}$ and $\mathrm{Fe}$ ions are clearly resolved. The spectrum above about $5 \mathrm{keV}$ is dominated by the $\mathrm{Fe}-\mathrm{K}$ line and the continuum, the latter of which has been used to determine the electron temperature. Similiar spectra are available for Cas-A,

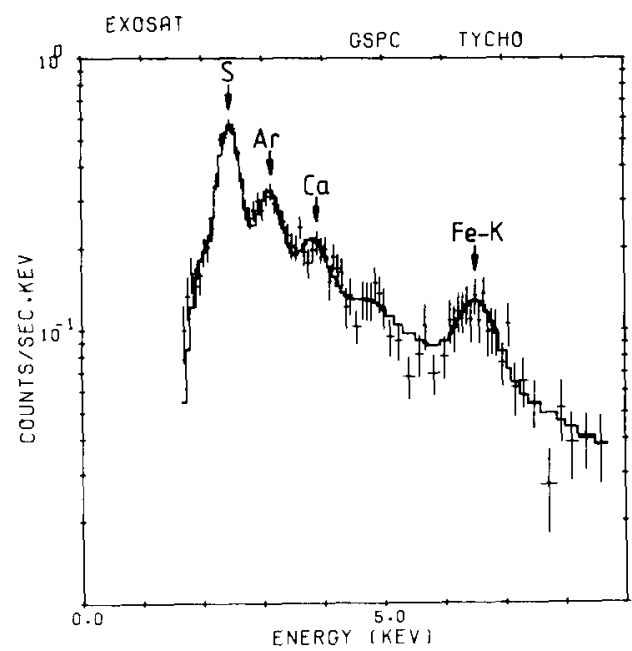

Fig. 3. EXOSAT GSPC spectrum of Tycho. The fit assumes a thermal bremsstrahlung continuum of $\mathrm{kT}=6.5 \mathrm{keV}$ and emission lines of $\mathrm{S}, \mathrm{Ar}, \mathrm{Ca}$ and $\mathrm{Fe}$ superimposed (from Smith et al. 1987).
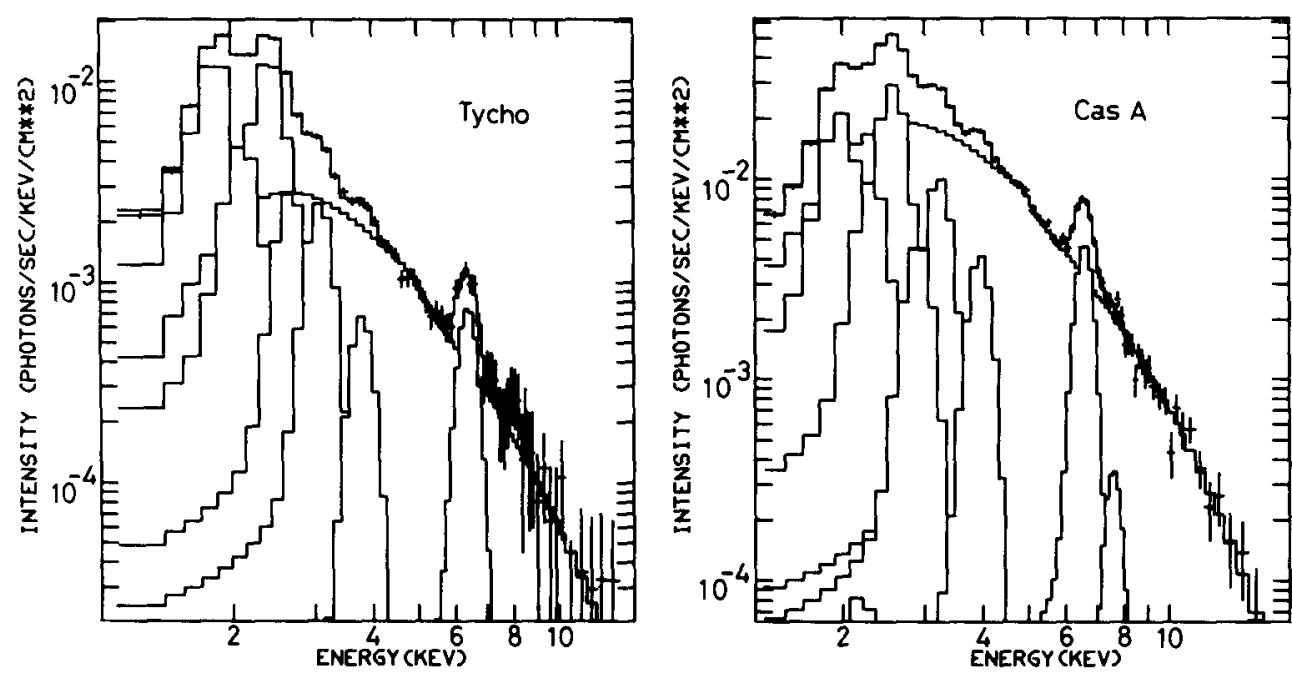

Fig. 4. Tenma GSPC spectrum of Cas-A and Tycho. The best fit assumes a single continuum spectrum and emission from 8 lines. The relative contribution of each component is shown in the lower histograms (from Tsunemi et al. 1986). 
Kepler, RCW 103 and $W 49 B$ and a detailed account of the EXOSAT spectra is given by Smith (1987). Tsunemi et al. (1986) have published the Tenma GSPC energy spectra of Cas-A and Tycho which extend to some lower energies to include the $\mathrm{Si}-\mathrm{K}$ lines (see figure 4).

The emission from shell type remnants is considered to originate from an optically thin plasma, which has been heated to X-ray temperatures by shock waves. Model fits to the measured spectra attempt to determine Xray temperature, total emission measure and elemental abundances, from which the density of the pre-shocked interstellar medium, the total sweptup mass and the explosion energy of the supernova can be derived using some 3-dimensional geometry and hyrodynamical solution for the expansion. If the contributions from the interstellar medium and the stellar ejecta can be disentangled, density and mass of the ejecta can be determined. In this way a link from the remnant to the progenitor star and the supernova type can be established in addition to the shock wave physics research. Early fits to the spectra have been based on the following assumption:

1. the emitting plasma is in collisional equilibrium ionization;

2. there is thermal equilibrium between ions and electrons implying the same temperature for both;

3. the electrons have a Maxwellian velocity distribution;

4. the plasma parameters including temperature, abundances, ionization stages, etc. are homogeneous over the entire remnant, at least over the field of view of the instrument;

5. the hydrodynamical evolution of the remnants can be described by the self similarity Sedov solution, and

6. the remnant expands into an ambient homogeneous medium.

The general results of these early analyses, which hold for almost any well studied remnant, can be summarized as follows:

1. The spectrum is well described by the superposition of the emission from a two component plasma characterized by a low temperature of about $0.2-0.5 \mathrm{keV}$ and a high temperature of about a few $\mathrm{keV}$;

2. a third, very high temperature component of about $30 \mathrm{keV}$ has been suggested to be present in Cas-A (Pravdo and Smith 1979) which is, however, inconsistent with the EXOSAT data (Jansen et al. 1987). For Tycho, Pravdo and Smith derive a similarly high temperature component which could not be confirmed by EXOSAT because of inadequate sensitivity (Smith et al. 1987);

3. elemental abundances from oxygen burning nucleosynthesis products including $\mathrm{Si}, \mathrm{S}, \mathrm{Ar}$ and $\mathrm{Ca}$ are largely overabundant compared with solar or cosmic values, whereas $\mathrm{Fe}$ tends to be less than solar;

4. even young remnants contain a large X-ray emitting mass. For instance, Fabian et al. (1980) have found more than 15 solar masses in Cas-A, and Reid et al. (1982) estimate 15 solar masses for Tycho as well.

The validity of each of the six assumptions listed above was questioned, very early on, mainly from theoretical arguments. Now, there is growing observational evidence supporting more or less substantial modifi- 
cations of the assumptions with subsequent revision of the parameters derived, the amount of which depends on age and environment of the individual remnant.

As pointed out by Gorenstein et al. (1974) the plasma in supernova remnants may not be in collisional ionization equilibrium because the time scale to ionize the heavy elements to the equilibrium level by electron collisions is of the order of $10^{4} /$ ne years (Canizares 1984). For electron densities ne of about $1-10 \mathrm{~cm}^{-2}$, this is large compared with the age of young remnants and has the effect that the heavy elements are underionized compared with their equilibrium population. Direct observational evidence for non equilibrium ionization (NEI) has been obtained from the analysis of the high resolution FPCS spectra of regions in Puppis-A (Canizares et al. 1983, Winkler et al. 1983, Fischbach et al. 1987) and in Cas-A (Markert et al. 1987). The NEI conditions are derived from the weakness of the forbidden lines relative to the resonance lines of OVII and NeIX (see figure 2). Vedder et al. 1986 has recently published the FPCS spectrum taken in the northern bright region of the Cygnus Loop (figure 5). From a $3 \sigma$ upper limit of the forbidden to resonance line ratio of OVII they conclude that even in a remnant as old as the Cygnus Loop at least sections exist within which collision equilibrium ionization has not been reached. However, Gabriel et al. (1985) have pointed out that fast electrons in an otherwise thermal plasma can mimic NEI conditions because they tend to excite preferentially the resonance line, and therefore a weak forbidden-toresonance line ratio may not be conclusive. They compute that $20 \mathrm{keV}$ electrons having a proportion of $1 \%$ of the total number of electrons are sufficient to explain the OVII lines as observed in Puppis-A.

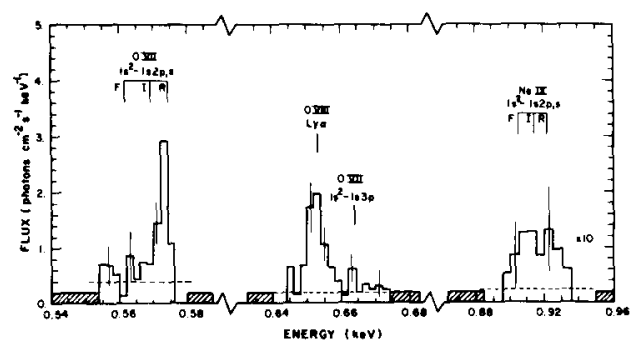

Fig. 5.Portions of the X-ray spectrum of the northern bright region of the Cygnus Loop measured with the FPCS (from Vedder et al. 1986).

Further evidence for NEI conditions in Tycho and Cas-A has been obtained from the EXOSAT (Jansen et al. 1987, Smith et al. 1987) and Tenma (Tsunemi et al. 1986) observations of the Fe-K line. Both experiments have measured consistently a line energy significantly lower than the value expected from CEI which is based upon a temperature derived from the high energy continuum. Applying NEI models both experiments agree that Tycho is substantially more underionized than Cas-A, which is plausible from the higher density in Cas-A.

Less direct but nonetheless evidence for NEI is deduced from the fact that 
NEI models fit the observed X-ray spectra better than CEI models. NEI models have been constructed by numerous authors to describe time dependent ionization (Itoh 1977, 1979, Gronenschild and Mewe 1982, Shull 1982, Hamilton et al. 1983, Hamilton and Sarazin 1984, Nugent et al. 1984). The gross effect of NEI is an enhanced emission from lower ionization stages, which mimics a separate low temperature CEI plasma in addition to the temperature indicated by the high energy continuum. This is the reason that early X-ray spectra could be approximated by two temperature CEI models. However, with increasing better spectral resolution and broader energy coverage the fits became increasingly poorer. In order to explain the strong line emission from heavy elements observed in young remnants, a significant overabundance compared to solar values had to be adopted. Furthermore high emission measures implying high densities were needed to explain the high level of soft X-ray emission, which in turn led to high masses for the remnants. NEI models have recently been applied to some remnants with the result that the CEI based estimates about the total X-ray emitting mass have been refined. Using NEI emission to model the surface brightness distribution observed with the Einstein HRI, Gorenstein et al. (1983) derive a total of about 4 solar masses for Tycho shared one to one by the ejecta and swept up matter. This has to be contrasted with the result of about 15 solar masses which Reid et al. (1982) deduced from the Einstein IPC image and a CEI model. An even lower value of only 0.6 solar masses for the X-ray emitting mass of Tycho has been presented by Tsunemi et al. (1986), which they conclude from a NEI analysis of the Tenma energy spectrum. Figure $4 \mathrm{~b}$ shows the excellent fit. Although the mass has come down significantly the heavy elements are still a factor of 6-15 overabundant - but including iron - compared with solar values. The Tenma spectrum of Cas-A has also been fitted acceptably with a single component NEI model with a remarkable low value of 2.4 solar masses and element abundances very close to solar values. The Tenma analysis, however, is in conflict with the EXOSAT results analyzed by Smith et al. (1987) and Jansen et al. (1987) who claim that the spectra cannot be explained by a single component but require a two component NEI model. This disagreement is most evident in the values given for the continuum temperature, which unfortunately affects the elemental abundances and masses strongly. Thus, Smith et al. quote 3.3 solar masses for the swept up mass in Tycho. It remains to be seen how this conflict will be resolved in the future. This is interesting, since the Tenma results, for the first time, are clearly in line with respect to element abundances and mass with what is currently predicted from supernova explosion models advocating a type I for Tycho and type II for Cas-A.

Hamilton et al. (1986 b) have recently re-analysed a collection of spectra of Tycho which have been obtained with the instruments preceding Tenma and EXOSAT. They assume a two component NEI model, one each for the blast wave and the reverse shock. The composition is taken to be solar for the uniform interstellar medium and of pure heavy elements for the ejecta which are stratified according to atomic number. From the spectra which are shown in figure 6 they conclude that the X-ray emission is dominated by the shocked ejecta with an important contribution from the blast wave at high energies. The total ejected mass is 1.4 solar masses out of which 
0.4 solar masses have not yet been shocked, and the mass shocked by the blast wave amounts to 1.3 solar masses. This is close to the results of Gorenstein et al. but significantly higher than the Tenma data analysis has shown. Hamilton et al. (1986 a) have successfully used this type of model to reproduce the featureless power-law like spectrum of SN1006 by putting SN1006 into a much lower ionization age than Tycho.
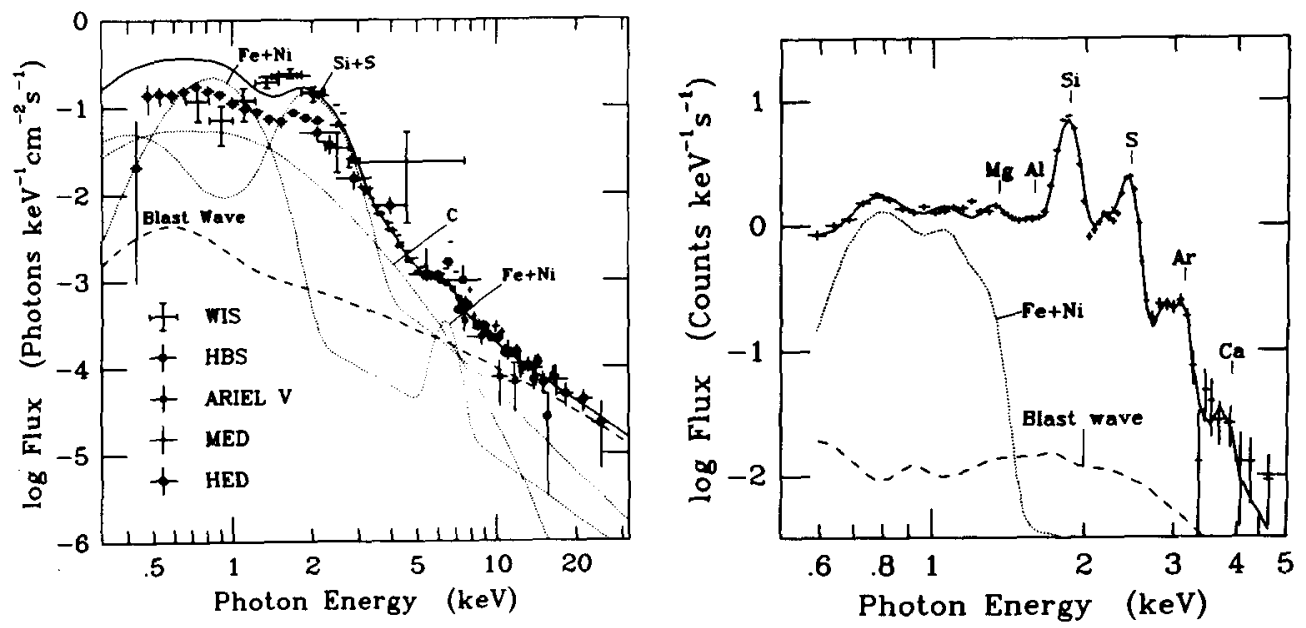

Fig. 6. Best fit of the two component NEI models of Hamilton et al. (1986) to low resolution (left) and high resolution (right) spectra of Tycho's supernova remnant.

The NEI models discussed above assume that the hydrodynamic evolution of the remnant can be described by the self similarity solutions of the Sedor type. Hughes and Helfand (1985) have instead used a numerical hydrodynamic shock code into which the time dependent ionization equations have been incorporated. Thus, the time dependent ionization structure can be computed simultaneously in both the blast wave heated ambient plasma as well as the reverse shock heated ejecta. Interestingly, they have found that the surface brightness distributions and the spectra of Kepler's supernova remnant as measured with the Einstein observatory instruments can be equally well reproduced if the emission originates predominantly either from the heated interstellar medium with the remnant in the Sedov phase or from the heated ejecta. Figure 7 shows the best fit to the Einstein SSS spectrum which is achieved with over-solar abundances of $\mathrm{Si}$ and $\mathrm{S}$ but under-solar abundance of $\mathrm{Fe}$. The over-abundance is more pronounced for the reverse shock case but it is lower, by a factor of 2 to 3, than the results of two temperature CEI models of Becker et al.(1980). Whereas the Sedov case is compatible with a type I supernova event, the reverse shock heated ejecta model requires an ejecta mass of about 4.5 solar masses, which implies a massive progenitor star. It would certainly be useful to further constrain the model and include into the analysis the high energy spectrum to beyond the Fe-K line, which is now available from EXOSAT. 


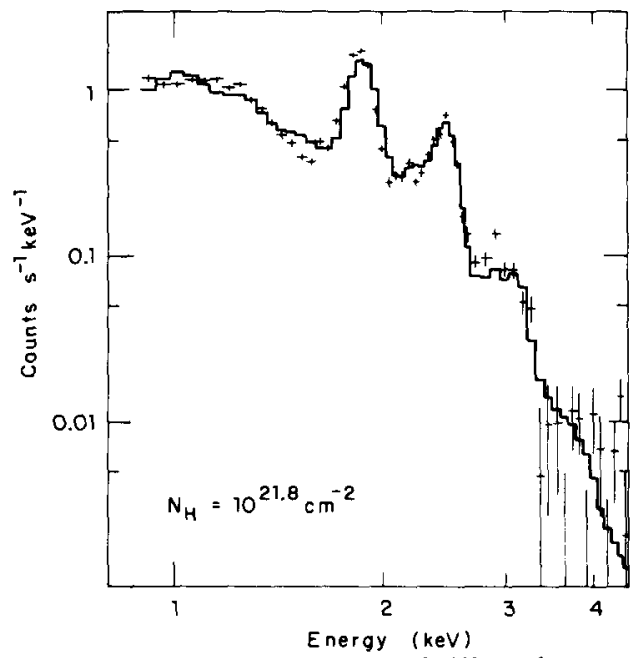

Fig. 7. Best fit of the hydrodynamic shock NEI model of Hughes and Helfand (1985) to the Einstein SSS spectrum of Kepler's supernova remnant.

In summary, spectral modelling has made progress in recent years by involving NEI in particular and better fits have been obtained with generally lower masses and lower elemental abundances have been obtained compared with CEI models. However, there seems to be no conclusion on the number of temperature components present in young remnants. It is striking that the analysis of relatively narrow band spectra like that by Tsumeni et al. for Tycho and Cas-A and that by Hughes and Helfand for Kepler favour a dominant single component. In contrast to this, the analysis of broad band spectra like that of Jansen et al. for Cas-A and Smith et al. for Tycho and that of Hamilton et al. for Tycho as well clearly require two NEI components which are associated with plasma heated by the blast wave and the reverse shock.

Spectrally resolved images: Spectrally unresolved images obtained with the Einstein HRI or IPC, or the EXOSAT CMA instrument, or even the earlier collimated scanning counters demonstrated a great deal of spatial structure to be present in many remnants. Structure like this may originate from different kinds of variations across the remnant, such as those of emission measure, i.e. density and depth of line of sight within the remnant, temperature, ionization structure, and even interstellar absorption column density towards remnants of significant extent. Clearly, these effects are important to be considered when determining elemental abundances and Xray emitting mass, and therefore a spectrum spatially integrated over the whole remnant or over substantial portions of the remnant is of limited information (see for instance Brinkmann and Fink 1987).

Spectral variations across the remnant of Cas-A were first discovered in the Einstein IPC data, which show significantly different pulse height spectra in the south-west and the north- east section (Murray et al. 1979). Using the brightness distribution observed with the HRI, a two-circularshell geometry and assuming pressure equilibrium, Fabian et al. (1980) has constructed a CEI temperature and density map revealing considerable variations within the remnant. In collaboration with the Naval Research Laboratory, the MPI group has obtained a spectrally resolved image on a sounding rocket flight (Aschenbach 1985). Using CEI emission and the 
element abundance of Becker et al. (1979), a temperature map has been derived, which confirms and expands the Einstein IPC results. The faint south-west region has a temperature in excess of $4 \mathrm{keV}$, whereas the bright northeast part is between 0.3 and $1 \mathrm{keV}$. The overall temperature distribution shows two distinct peaks at $0.5 \mathrm{keV}$ and $5.4 \mathrm{keV}$. The hot component forms an almost complete shell along the outermost boundary at a radial distance of about 3 arcmin from the centre. The interior is rather uniform at the low temperature level. Assuming two spherical shells for the emission region, density and pressure maps have been constructed and the remnant is not found in pressure equilibrium, but with the highest pressure occurring along the outer boundary. Interestingly, the interior which seems to be in pressure equilibrium is separated from the outermost annulus by an about 30 arcsec wide pressure minimum, indicating a deceleration of the blast wave.

The EXOSAT imaging telescopes have been used to take very long exposures of Cas-A and to measure the spectral variations with high statistical accuracy, but with proportional counter type resolution (Jansen et al. 1987). They also find significant CEI temperature variations with the highest temperatures occurring in an outer annulus about 3 arcmin from the centre. Analysing the temperature distribution in radial sectors, the high temperature component is most pronounced in the smooth and faint regions whereas in the clumpy and bright regions the high temperature component appears reduced. Figure 8 shows two examples of temperature profiles reproduced from the paper of Jansen et al.
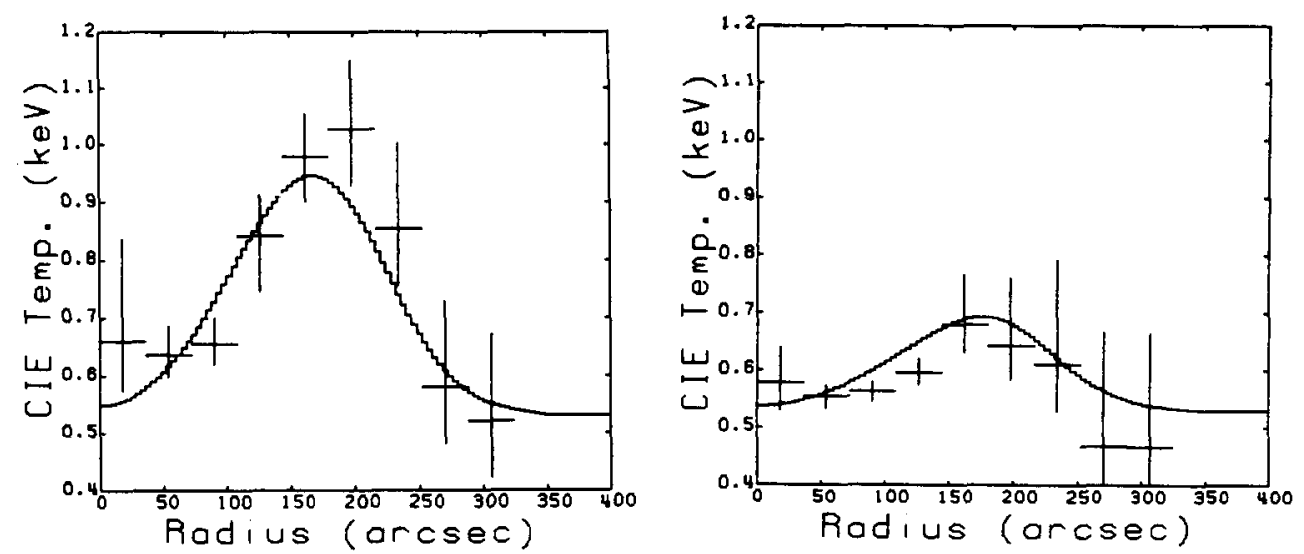

Fig. 8. CEI temperature profiles of the smooth and faint west south-west portion of Cas A (left) and the clumpy and bright east south-east portion of Cas-A (right) as deduced from the EXOSAT PSD images by Jansen et al. (1987).

The Einstein IPC, the MPI rocket and the EXOSAT PSD results largely agree on the gross temperature distribution and demonstrate two temperature components in Cas-A one of which is apparently associated with the blast wave indicated by its high temperature, its outermost location and the underlying smooth and faint brightness distribution. As expected the 
low temperature component is associated with the reverse shock, which has heated the clumpy ejecta located more inside the remnant. So even in a remnant as young as Cas- $A$ significant emission from heated ejecta has been found and this has independently been established by the remnant integrated spectra like those from the SSS, EXOSAT and Tenma as well as the spectrally resolved, but low energy images.

The first spectrally resolved images of the middle-aged remnant Puppis-A have been reported by the MPI group from a sounding rocket experiment (Pfeffermann et al. 1980). The CEI temperature map with a resolution of 3 arcmin shows a rather uniform temperature of $2-5.10^{6} \mathrm{~K}$ for the interior, which agrees fairly well with the results from the Einstein high resolution FPCS CEI analysis (Winkler et al. 1981 a, b). In a subsequent paper, however, Canizares et al. (1983) showed that the spectrum taken with the 3

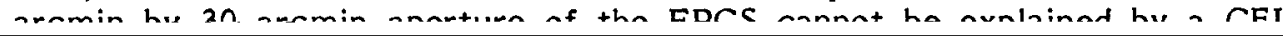

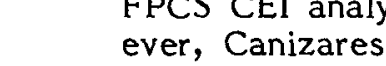


reported $C C D$ images of the region of the eastern knot in the forbidden red and green coronal iron lines, and under the assumption that the knot, which is supposed to be an interstellar cloud, is in CEI at a temperature of $\log T=6.35$ they derive a minimum mass of about 0.1 solar masses with a density in the range of 23 to $49 \mathrm{~cm}^{-3}$. The EXOSAT results show that there is significantly cooler gas in the knot region, and thus they do not support Teske's and Petre's view that the visible FeXIV emission is from cooler inclusions in a still hotter medium. A comparison of the EXOSAT CEI temperature map with the 6 arcmin diameter fields observed with the SSS and the 3 arcmin by 30 arcmin rectangular fields observed with the FPCS clearly reveals that the spectrum within each of the Einstein fields is not uniform but varies spatially (Jansen, Aschenbach and Bleeker, 1987), although these fields are already small compared with the extent of the remnants.

Spectrally resolved images have been taken also from the old Vela supernova remnant and the Cygnus Loop with the Einstein IPC. For the Cygnus Loop, $\mathrm{Ku}$ et al. (1984) have pointed out that CEI best fits show lower temperatures along the limb compared with the centre, and that temperatures anticorrelate with intensity as expected for approximate pressure equilibrium. This view has been supported by Charles et al. (1985) by a detailed spectral study of two 10 wide fields located at the southern and western boundary of the remnant. Temperature and emission measure variations on scales as small as 4 arcmin have been found, favouring the presence of an inhomogeneous cloudy interstellar medium. Similar results have been obtained for the Vela remnant by Kahn et al. (1985), although the two remnants different significantly in their overall morphological appearance. However, the observations do not preclude pressure variations as large as a factor of 10 .

Future prospects: Since the early years of proportional counters much progress has been made in supernova remnant research due to the availability of high resolution imaging, high resolution spectroscopy and broad band energy coverage. High resolution imaging made visible the cloudy interstellar medium and the clumpy ejecta, and it made possible the first comparative investigations between $\mathrm{X}$-ray, optical and radio morphology; in particular the first detailed observational studies of shocked clouds and possible thermal evaporation. The Einstein FPCS and SSS have shown that nonequilibrium ionization may be present in many remnants, even as old as the Cygnus Loop. However, it seems that the present generation of NEI models does not explain the broad band, high energy spectra as observed by EXOSAT for instance, unless multi NEI models with a sufficient number of free parameters are used. In order to better understand the images and spectra, hydrodynamical shock codes coupled with time dependent ionization are needed with an improved knowledge of the electron energy distribution which determines the ionization structure. These model calculations should take into account the results from the supernova explosion simulations including velocity, density and elemental abundances of the debris to predict in detail the effects of the progenitor star. Similarly, the ambient medium into which the remnant expands has to be considered, including a stellar wind of the progenitor star, and a multi-component medium. 
A key issue for further progress from an observational point of view is the future availability of spectrally resolved images which cover the energy region from 0.3 to about $10 \mathrm{keV}$. An energy band as broad as this is needed to disentangle the emission from the low temperature ejecta as well as from the high temperature interstellar medium heated by the blast wave and to determine independently elemental abundances for each component. A spectral resolving power of about 50 is adequate. The spatial resolution required is of course a small fraction of the remnant size. Hydrodynamical NEI model calculations of Brinkmann and Fink (1987) suggest that for a young remnant like Tycho a resolution of about 20 arcsec is adequate to resolve the ionization structure. Taking the degree of clumpiness, even lower values are indicated. For older remnants like Puppis-A, Vela or the Cygnus Loop, an angular resolution approaching the small structural scales present in the cloudy interstellar medium is appropriate. The bright eastern knot in Puppis-A for instance has a size of about 1 arcmin.

At present there are 5 missions being planned which carry imaging telescopes, i.e. the German ROSAT (1990) and Spectrosat (1993) missions, the Italian SAX (1992) mission, NASA's AXAF (1995) and XMM (1998) of the European Space Agency. The numbers in parenthesis give the currently envisaged launch dates. Except ROSAT, all other four missions await final approval. ROSAT will take images with high angular resolution ( 5 arcsec), and extremely high contrast due to the steep telescope point spread function and almost vanishing scattering. This will allow a search for compact objects within remnants at an significantly increased level of sensitivity compared with Einstein. With a throughput about 8 times greater than the Einstein HRI and a comparable background level, remnants can be studied to lower surface brightness within and outside our galaxy. Spectrally resolved images with an angular resolution of 20 arcsec and a spectral resolving power of about 2.5 will be taken with the position sensitive proportional counter in the energy band $0.1-2.2 \mathrm{keV}$. This spectral resolution is insufficient to resolve individual lines and to perform detailed plasma diagnostics but it will establish spectral variations across many remnants at an unprecedented level of angular resolution, and it will constrain the nonthermal component in Crab-like and composite remnants. Spectrosat will be a ROSAT follow-up modified by a transmission grating which will increase the resolving power to about 50-100.

At present, SAX will be the first mission which will carry imaging telescopes working up to $10 \mathrm{keV}$, with a spatial resolution of about 1 arcmin and a resolving power of about 10 at the Fe-K line. Very few years before the turn of the millenium, hopefully, the two great observatories AXAF and XMM will be launched into orbit. Both observatories cover the energy band from 0.1 to $10 \mathrm{keV}$. With AXAF sub-arcsecond imaging will become possible and very high spectral resolution as well, although at moderate throughput. XMM will perform low to high spectral resolution observations with high throughput but at the expense of angular resolution ( $30 \mathrm{arcsec}$ ).

Acknowledgements: I like to thank Dr. D. Helfand, F.A. Jansen, and Drs. A. Smith and H. Tsunemi, who provided me with reprints, preprints and data partially prior to publication. 


\section{References}

Aschenbach, B., Sp.Sc.Rev. 40, 447 (1985).

Aschenbach, B. and Brinkmann, W., Astron. Astrophys. 41, 147 (1975).

Becker, R.H., Boldt, E.A., Holt, S.S., Serlemitsos, P.J., White, N.E., Ap.J. 237, $L 77$ (1980).

Becker, R.H., Holt, S.S., Smith, B.W., White, N.E., Boldt, E.A., Mushotzky, R.F., Serlemitsos, P.J. Ap.J. 234, L73 (1979).

Brinkmann, W. and Aschenbach, B., Nature 313, 662(1986).

Brinkmann, W. and Fink, H.H., this volume (1987).

Canizares, C.R., Proc. of the Intern. Symp. on "X-ray Astronomy '84", Oda, M. and Giacconi, R., eds., Bologna, 275 (1984).

Canizares, C.R., Winkler, P.F., Markert, T.H., Berg, C., "Supernova Remnants and their X-ray Emission", IAU Symp. No. 101, Danziger, J. and Gorenstein, P., eds., (D. Reidel), 205 (1983).

Charles, P.A., Kahn, S.M., McKee, C.F., Ap.J. 295, 456 (1985).

Cowie, L.L. and McKee, C.F., Ap.J. 211, 135 (1977).

Danziger, J. and Gorenstein, P., "Supernova Remnants and their X-ray Emission", Proc. of the IAU Symp. No. 101, Venice (D. Reidel) 1983.

Davelaar, J. and Smith, A., Proc. of the George Mason University workshop on "The Crab Nebula and Related Objects", Kafatos, M.C. and Henry, R.B.C., eds., Fairfax (Cambridge University Press), 219 (1986).

Fabian, A.C., Willingale, R., Pye, J.P., Murray, S.S., Fabbiano, G., M.N.R.A.S. 193, 175 (1980).

Fischbach, K.F., Canizares, C.R., Markert, T.H., Coyne, J.H., this volume (1987).

Gabriel, A.H., Acton, L.W., Bely-Dubau, F., Faucher, P., Proc. ESA Workshop on "Cosmic X-Ray Spectroscopy Mission", ESA SP-239, 137 (1985).

Gorenstein, P., Harnden, Jr., F.R., Tucker, W.H., Ap.J. 192, 661 (1974).

Gorenstein, P., Seward, F., Tucker, W., "Supernova Remnants and their Xray Emission", IAU Symp. No. 101, Danziger, J. and Gorenstein, P., eds., (D. Reidel), 1, 1983.

Gronenschild, E.H.B.M. and Mewe, R., Astron. Astrophy. Suppl. 48, $305^{*}$ (1982).

Hamilton, A.J.S., Sarazin, C.L., Chevalier, R.A., Ap.J. Suppl. 51, 115 (1983).

Hamilton, A.J.S. and Sarazin, C.L., Ap.J. 284, 601 (1984).

Hamilton, A.J.S., Sarazin, C.L., Szymkowiak, A.E., Ap.J. 300, 698 (1986a). Hamilton, A.J.S., Sarazin, C.L., Szymkowiak, A.E., Ap.J. 300, 713 (1986b). Heiles, C., Ap.J. 140, 470 (1964).

Helfand, D.J., (1987) this conference, not published.

Helfand, D.J. and Becker, R.H., Nature 307, 215 (1984).

Hester, J.J. and Cox, D.P., Ap.J. 300, 675 (1986).

Holt, S.S., "Supernova Remnants and their X-ray Emission", IAU Symp. No.

101, Danziger, J. and Gorenstein, P., eds., (D. Reidel), 17 (1983).

Hughes, J.P. and Helfand, D.J., Ap.J. 291, 544 (1985).

Itoh, H., Publ. Astron. Soc. Japan 29, 813 (1977).

Itoh, H., Publ. Astron. Soc. Japan 31, 541 (1979).

Jansen, F.A., Smith, A., Bleeker, J.A.M., de Korte, P.A.J., Peacock, A., White, N.E., submitted to Ap.J. (1987).

Jansen, F.A., Aschenbach, B., Bleeker, J.A.M., this volume (1987). 
Kahn, S.M., Gorenstein, P., Harnden, Jr., F.R., Seward, F.D., Ap.J. 299, 821 (1985).

Ku, W.H.-M., Kahn, S.M., Pisarski, R., Long, K.S., Ap.J. 278, 615 (1984).

Markert, T.H., Blizzard, P.L., Canizares, C.R., Hughes, J.P., this volume (1987).

Mathewson, D.S., Ford, V.L., Tuohy, I.R., Mills, B.Y., Turtle, A.J., Helfand, D.J., Ap.J. Suppl. 58, 197 (1985).

McKee, C.F., Ap.J. 188, 335 (1974).

McKee, C.F., "Supernova: A Survey of Current Research", Proc. NATO ASI Cambridge, Rees, M. and Stoneham, R.J., eds., (D. Reidel), 433 (1981).

Murray, S.S., Fabbiano, G., Fabian, A.C., Epstein, A., Giacconi, R., Ap.J. 234, L69 (1979).

Nomoto, K. and Tsuruta, S., Ap.J. 305, L19 (1986).

Nugent, J.J., Pravdo, S.H., Garmire, G.P., Becker, R.H., Tuohy, I.R., Winkler, P.F., Ap.J. 284, 612 (1984).

Pacini, F. and Salvati, M., Ap.J. 186, 249 (1973).

Petre, R., Canizares, C.R., Kriss, G.A., Winkler, P.F., Ap.J. 258, 22 (1982).

Pfeffermann, E., Aschenbach, B., Bräuninger, H., Heinecke, N., Ondrusch,

A., Trümper, J., Bull. Am. Astron. Soc. 11, 789 (1980).

Pravdo, S.H. and Smith, B.W., Ap.J. 234, L195 (1979).

Rees, M.J. and Gunn, J.E., M.N.R.A.S. 167, 1 (1974).

Reid, P.B., Becker, R.H., Long, K.S., Ap.J. 261,485 (1982).

Reynolds, S.P. and Chevalier, R.A., Ap.J. 245, 912 (1981).

Reynolds, S.P. and Chevalier, R.A., Ap.J. 278, 630 (1984).

Serlemitsos, P.J., Boldt, E.A., Holt, S.S., Ramaty, R., Brisken, A.F., Ap.J. 184, L1 (1973).

Seward, F.D., Comm. Astroph. XI, 1, 15 (1985).

Smith, A., Davelaar, J., Peacock, A., Taylor, B.G., Morini, M., Robba, N.R., submitted to Ap.J. (1987).

Smith, A., this volume (1987).

Shull, J.M., Ap.J. 262, 308 (1982).

Szymkowiak, A.E., NASA Techn. Memor. 86169 (1985).

Teske, R.G. and Petre, R., Ap.J. 314, 673 (1987).

Tsunemi, H., Yamashita, K., Masai, K., Hayakawa, S., Koyama, K., Ap.J. 306, 248 (1986).

Vedder, P.W., Canizares, C.R., Markert, T.H., Pradhan, A.K., Ap.J. 307, 269 (1986).

Weiler, K., The Observatory 103, 85 (1983).

Winkler, P.F., Canizares, C.R., Clark, G.W., Markert, T.H., Petre, R., Ap.J. 245, 574 (1981a).

Winkler, P.F., Canizares, C.R., Clark, G.W., Markert, T.H., Kalata, K., Schnopper, H.W., Ap.J. 246, L27 (1981b).

Winkler, P.F., Canizares, C.R., Bromley, B.C., "Supernova Remnants and their X-ray Emission", IAU Symp. No. 101, Danziger, J. and Gorenstein, P., eds., (D. Reidel), 245 (1983). 